\title{
Measuremental Data: Seven Measures of Central Tendency
}

\author{
Dhritikesh Chakrabarty * \\ Department of Statistics, Handique Girls' College, Guwahati, Assam, India
}

\begin{abstract}
Recently, four measures of central tendency namely Arithmetic-Geometric Mean (AGM), Arithmetic-Harmonic Mean (AHM), Geometric-Harmonic Mean (GHM) and ArithmeticGeometric-Harmonic (AGHM) have been derived from the three Pythagorean means namely Arithmetic Mean (AM), Geometric Mean (GM) and Harmonic (HM). An attempt has here been made on establishing each of these four measures of average as a measure of central tendency of measuremental data. This paper is based on a brief description on the seven measures namely AM, GM, HM, AGM, AHM, GHM \& AGHM of central tendency of data.
\end{abstract}

Keywords : Central Tendency, Measure, AGM, AHM, GHM, AGHM

\section{Introduction}

Average [Bakker, 2003] is a concept which describes any characteristic of an aggregate / population / class of individuals overall but not of an individual in the aggregate / population / class in particular. It is used in most of the measures associated to data (or list of numerical values). Pythagoras [Riedweg, 2005], one exponent of mathematics, defined the three most common averages namely arithmetic mean (AM), geometric mean (GM) and harmonic mean (HM) which were given the name "Pythagorean Means" [Chakrabarty, 2016b; De Carvalho, 2016; Kolmogorov, 1933] as a mark of honor to him. Later on, a number of definitions/ formulations of average like Quadratic Mean, Square Root Mean , Cubic Mean, Cube Root Mean, Generalized p Mean \& Generalized $p^{t h}$ Root Mean etc. in addition to AM, GM \& HM had been derived due to necessity of handling different situations [Chakrabarty, 2016a, 2018c, 2019f, 2020d; De Carvalho, 2016; Kolmogorov, 1930].

The next trend was towards the development of generalized definitions of average namely Generalized f-Mean [Chakrabarty, 2018a,f], Generalized $f_{H}$-Mean [Chakrabarty, 2018b] chakrabarty2018four and Generalized $f_{G}$-Mean [Chakrabarty, 2018d, 2019d,e] and general method of defining average [Chakrabarty, 2019a,b].

In statistics, the three Pythagorean means [Chakrabarty, 2021e] are used in measuring the central tendency of numerical data [Plackett, 1958; Weisberg and Weisberg, 1992; Williams, 1984]. However, the accuracy of the value of central tendency yielded by each of the three Pythagorean means is not known. Recently, there have been a lot of studies on analysis of numerical data based on average in general and on Pythagorean means specially [Chakrabarty, 2014a,b,c, 2015a,b,c,d,e,f,g, 2016a, 2017a,b,c, 2018e, 2019c] . In the mean time, several attempts have been made on determining accurate value of central tendency of numerical data. However, still there is necessity of more accurate measure of the same. With an objective of finding out more accurate measure of central tendency of data, four measures of central tendency namely Arithmetic-Geometric Mean (AGM) [Chakrabarty, 2020f, 2021b,c,d; David,

*corresponding author:dhritikesh.c@rediffmail.com ,dhritikeshchakrabarty@gmail.com 
2004; Hazewinkel, 2001; Kolmogorov, 1933], Arithmetic-Harmonic Mean (AHM) [Chakrabarty, 2020a,b, 2021b; Foster and Phillips, 1984], Geometric-Harmonic Mean (GHM) [Chakrabarty, 2020e, 2021b] and Arithmetic-Geometric-Harmonic (AGHM) [Chakrabarty, 2020c, 2021a,b,f,g,h,i] have been derived from the three Pythagorean means. An attempt has here been made on establishing each of these four measures of average as a measure of central tendency of measuremental data. This paper is based on a brief description on the seven measures namely AM, GM, HM, AGM, AHM, GHM \& AGHM of central tendency of data.

\section{Measurement of average}

The seven measures of average considered here are Arithmetic Mean (AM), Geometric Mean (GM), Harmonic Mean (HM), Arithmetic-Geometric Mean (AGM), Arithmetic-Harmonic Mean (AHM), GeometricHarmonic Mean (GHM) and Arithmetic-Geometric-Harmonic (AGHM).

Let

$$
x_{1}, x_{2}, \ldots \ldots \ldots, x_{N}
$$

be $\mathrm{N}$ positive numbers or values or observations (not all equal or identical) which are strictly positive.

If $a_{0}, g_{0}$ and $h_{0}$ are respectively the $\mathrm{AM}$, the $\mathrm{GM} \&$ the $\mathrm{HM}$ of these $\mathrm{N}$ numbers (or values or observations) then

$$
\begin{gathered}
a_{0}=A M\left(x_{1}, x_{2} \ldots \ldots x_{N}\right)=\frac{1}{N} \sum_{i=1}^{N} x_{i} \\
g_{0}=G M\left(x_{1}, x_{2} \ldots \ldots x_{N}\right)=\left(\prod_{i=1}^{N} x_{i}\right)^{\frac{1}{N}} \\
h_{0}=H M\left(x_{1}, x_{2} \ldots \ldots x_{N}\right)=\left(\frac{1}{N} \sum_{i=1}^{N} x_{i}^{-1}\right)^{-1}
\end{gathered}
$$

Which satisfies the inequality

$$
\operatorname{Largest}\left(x_{1}, x_{2} \ldots \ldots x_{N}\right)>a_{0}>h_{0}>g_{0}>\operatorname{Smallest}\left(x_{1}, x_{2} \ldots \ldots x_{N}\right)[4,16,49,51]
$$

\section{$2.1 \quad$ AGM}

Let the two sequences $\left\{a_{n}\right\}$ and $\left\{g_{n}\right\}$ be defined by

$$
a_{n+1}=\frac{1}{2}\left(a_{n}+g_{n}\right)
$$

and ,

$$
g_{n+1}=\left(a_{n} \times g_{n}\right)^{\frac{1}{2}}
$$

respectively where the square root takes the principal value. Then, the two sequences $\left\{a_{n}\right\}$ and $\left\{g_{n}\right\}$ converge to a common point $M_{A B}$. This common point of convergence can be termed as the ArithmeticGeometric Mean of $\left(x_{1}, x_{2} \ldots \ldots x_{N}\right)$.

Accordingly AGM can be defined as foolows:

\section{Defination of AGM:}

The Arithmetic-Geometric Mean of the $N$ positive real numbers $\left(x_{1}, x_{2} \ldots \ldots x_{N}\right)$ denoted by $A G M\left(x_{1}, x_{2} \ldots \ldots x_{N}\right)$ is the common limit (or equivalently the common point of convergence) $M_{A G}$ of the two common sequences $\left\{a_{n}\right\}$ and $\left\{g_{n}\right\}$ defined by Eq. 5 and Eq. 6respectively where the square root takes the principal value and $a_{0} \& g_{0}$ are defined by Eq. 1 and Eq. 2 respectively. 


\subsection{AHM}

Let $\left\{a_{n}^{/}\right\}$and $\left\{h_{n}^{/}\right\}$be two sequences defined by

$$
\begin{gathered}
a_{n+1}^{\prime}=\frac{1}{2}\left(a_{n}^{\prime}+b_{n}^{\prime}\right) \\
h_{n+1}^{\prime}=\left[\frac{1}{2}\left\{\left(a_{n}^{\prime}\right)^{-1}+\left(b_{n}^{\prime}\right)^{-1}\right\}\right]^{-1}
\end{gathered}
$$

repectively. Then, the two sequences $\left\{a_{n}^{\prime}\right\}$ and $\left\{h_{n}^{\prime}\right\}$ converses to the same pont $M_{A H}$ This common point of convergence $M_{A H}$ can be termed as the Arithmetic-Harmonic Mean of $\left(x_{1}, x_{2} \ldots \ldots x_{N}\right)$.

Accordingly, AHM can be defined as follows:

\section{Defination of AHM:}

The Arithmetic-Harmonic Mean of the $N$ positive real numbers $\left(x_{1}, x_{2} \ldots \ldots x_{N}\right)$ denoted by $A H M\left(x_{1}, x_{2} \ldots \ldots x_{N}\right)$ is the common limit (or equivalently the common point of convergence $M_{A H}$ of two sequences $\left\{a_{n}^{\prime}\right\}$ and $\left\{h_{n}^{\prime}\right\}$ defined respectively by Eq. 7 and Eq. 8 where $a_{0} \& h_{0}$ are defined by Eq. 1 and Eq. 3 respectively.

\subsection{GHM}

Let $\left\{g^{/ /}\right\}$and $\left\{h^{/ /}\right\}$be two sequences defined respectively by

$$
\begin{gathered}
g_{n+1}^{/ /}=\left(g_{n}^{/ /} \times h_{n}^{/ /}\right)^{\frac{1}{2}} \\
h_{n+1}^{/ /}=\left[\frac{1}{2}\left(g_{n}^{/ /}\right)^{-1}\left(h_{n}^{/ /}\right)^{-1}\right]^{-1}
\end{gathered}
$$

where the square cube takes the principal value.

The, the two sequences $\left\{g^{/ /}\right\}$and $\left\{h^{/ /}\right\}$converges to the same point $M_{G H}$. This common point of convergence $M_{G H}$.can be termed as the Geometric-Harmonic Mean of $\left(x_{1}, x_{2} \ldots \ldots x_{N}\right)$.

Accordingly, GHM can be defined as follows:

\section{Defination of GHM:}

The Geometric-Harmonic Mean (GHM) of the $N$ positive real numbers $\left(x_{1}, x_{2} \ldots \ldots x_{N}\right)$ denoted by $\operatorname{GHM}\left(x_{1}, x_{2} \ldots \ldots x_{N}\right)$ is the common limit (or equivalently the common point of convergence) $M_{G H}$ of two the two sequences $\left\{g^{/ /}\right\}$and $\left\{h^{/ /}\right\}$defined by Eq. 9 and Eq. 10 respectively where the square root takes the principal value and $g_{0} \& h_{0}$ are defined by Eq. 2 and Eq. 3 respectively.

\subsection{AGHM}

Let us define the three sequences $\left\{a_{n}^{/ / /}\right\},\left\{g_{n}^{/ / /}\right\},\left\{h_{n}^{/ / /}\right\}$defined by

$$
\begin{gathered}
a_{n}^{/ / /}=\frac{1}{3}\left(a_{n}^{/ / /}+g_{n}^{/ / /}+h_{n}^{/ / /}\right) \\
g_{n}^{/ / /}=\left(a_{n}^{/ / /} \times g_{n}^{/ / /} \times h_{n}^{/ / /}\right)^{\frac{1}{3}} \\
a_{n}^{/ / /}=\left[\frac{1}{3}\left\{\left(a_{n}^{/ / /}\right)^{-1}+\left(g_{n}^{/ / /}\right)^{-1}+\left(h_{n}^{/ / /}\right)\right\}\right]^{-1}
\end{gathered}
$$

respectively where the cube root takes the principal value.

Then, the three sequences $\left\{a_{n}^{/ / /}\right\},\left\{g_{n}^{/ / /}\right\},\left\{h_{n}^{/ / /}\right\}$converge to a common point $M_{A G H}$. This common point of convergence can be termed as the Arithmetic-Geometric-Harmonic Mean of $\left(x_{1}, x_{2} \ldots \ldots x_{N}\right)$.

Accordingly, AGHM can be defined as follows:

\section{Defination of GHM:}

The Arithmetic-Geometric-Harmonic Mean (AGHM) of the $N$ positive real numbers $\left(x_{1}, x_{2} \ldots \ldots x_{N}\right)$ denoted by $\operatorname{AGHM}\left(\left(x_{1}, x_{2} \ldots \ldots x_{N}\right)\right.$ is the common limit (or equivalently the common point of convergence) $M_{A G H}$ of the three sequences $\left\{a_{n}^{/ / /}\right\},\left\{g_{n}^{/ / /}\right\},\left\{h_{n}^{/ / /}\right\}$defined by Eq. 11, Eq. 12, Eq. 13 respectively where the cube root takes the principal value and $a_{0}, g_{0}, h_{0}$ are defined by Eq. 1, Eq. 2, Eq. 3. 


\section{Measure of Central Tendency of Data}

The seven measures of central tendency of data, measuremental in nature, discussed here are AM, GM,HM, AGM, AHM,GHM \& AGHM. Among these seven, the first three are already established and widely used ones while the other four have been attempted here. The logical background of the seven measures has been explained below:

\subsection{AM as a Measure of Central Tendency}

If $\mu$ is the central tendency of the observations of $\left(x_{1}, x_{2} \ldots \ldots x_{N}\right)$ then the observations are composed of $\mu$ and random errors.In other words, these can be described by or expressed as

$$
x_{i}=\mu+\epsilon_{i},(i=1,2, \ldots . . N)
$$

where $\epsilon_{1}, \epsilon_{2}, \ldots . . \epsilon_{N}$ are the random errors, which assume positive and negative values in random order, associated to $\left(x_{1}, x_{2} \ldots \ldots x_{N}\right)$ respectively.

In this case, $A M\left(x_{1}, x_{2} \ldots \ldots x_{N}\right) \rightarrow \mu$ as $N \rightarrow \infty$ where

$$
A M\left(x_{1}, x_{2} \ldots \ldots x_{N}\right)=\frac{1}{N} \sum_{i=1}^{N} x_{i}
$$

This implies that $\left(x_{1}, x_{2} \ldots \ldots x_{N}\right)$ can be regarded as a measure of the value of $\mu$ or equivalently the central tendency of the observations $\left(x_{1}, x_{2} \ldots \ldots x_{N}\right)$.

\subsection{GM as a Measure of Central Tendency}

Again since the observations $\left(x_{1}, x_{2} \ldots \ldots x_{N}\right)$ consists of $\mu$ and random errors, theses can be described by or expressed as

$$
x_{i}=\mu \epsilon_{i}^{\prime \prime},(i=1,2,3 \ldots . . N)
$$

where $\epsilon_{1}^{/ /}, \epsilon_{2}^{/ /}, \ldots . ., \epsilon_{N}^{/ /}$are the random errors, which assume values in $(0,1)$ and in $(1, \infty)$ in random order associate to $\left(x_{1}, x_{2} \ldots \ldots x_{N}\right)$ respectively. In this case, $G M\left(x_{1}, x_{2} \ldots \ldots x_{N}\right) \rightarrow \mu$ as $N \rightarrow \infty$ where

$$
G M\left(x_{1}, x_{2} \ldots \ldots x_{N}\right)=\left[\sum_{i=1}^{N} x_{i}\right]^{\frac{1}{N}}
$$

This implies that $G M\left(x_{1}, x_{2} \ldots \ldots x_{N}\right)$ can also be regarded as a measure of the value of $\mu$ or equivalently the central tendency of the observations $\left(x_{1}, x_{2} \ldots \ldots x_{N}\right)$.

\subsection{HM as a Measure of Central Tendency}

Again since the observations $\left(x_{1}, x_{2} \ldots \ldots x_{N}\right)$ consist of $\mu$ and random errors, therefore, the reciprocals $\left(x_{1}^{-1}, x_{2}^{-1} \ldots \ldots x_{N}^{-1}\right)$ are composed of $\mu^{-1}$ and random errors different from the respective random errors $\epsilon_{1}, \epsilon_{2}, \ldots \ldots \epsilon_{N}$ provided $\left(x_{1}, x_{2} \ldots \ldots x_{N}\right)$ are different from zero.

In this case thus,

$$
x_{i}^{-1}=\mu^{-1}+\epsilon_{i}^{\prime},(i=1,2, \ldots \ldots \ldots, N)
$$

where, $\left(\epsilon_{1}^{\prime}, \epsilon_{2}^{\prime}, \ldots . \epsilon_{N}^{\prime}\right)$ are the random errors, which assume positive and negative values in random order, associated to are the random errors associated to $\left(x_{1}^{-1}, x_{2}^{-1} \ldots \ldots x_{N}^{-1}\right)$ respectively. In this case, $H M\left(x_{1}, x_{2} \ldots \ldots x_{N}\right) \rightarrow \mu$ as $N \rightarrow \infty$ where

$$
H M\left(x_{1}, x_{2} \ldots \ldots x_{N}\right)=\left[\frac{1}{N} \sum_{i=1}^{N} x_{i}\right]^{-1}
$$

This implies that $H M\left(x_{1}, x_{2} \ldots \ldots x_{N}\right)$ can also be regarded as a measure of the value of $\mu$ or equivalently the central tendency of the observations $\left(x_{1}, x_{2} \ldots \ldots x_{N}\right)$. 


\subsection{AGM as a Measure of Central Tendency}

Since each of $a_{n} \& g_{n}$ is approximate value of $\mu$,

$a_{0}=\mu+\delta_{0}$ and $g_{0}=\mu+\xi_{0}$, for real numbers $\delta_{0}$ and $\xi_{0}$.

This implies, $\delta_{0}>\xi_{0}$ since $a_{0}>g_{0}$.

By the same logic,

$a_{n+1}=\mu+\delta_{n+1}$ and $g_{n+1}=\mu+\xi_{n+1}$, for real numbers $\delta_{n+1}$ and $\xi_{n+1}$.

Since $a_{n+1}$ is the AM of $a_{n}$ and $g_{n}$

Therefore, $a_{n}>a_{n+1}>g_{n}$

Which implies, $\delta_{n}>\delta_{n+1}$, i.e., sequence $\left\{\delta_{n}\right\}$ is decreasing.

This implies, $\delta_{n}<\delta_{0}$, i.e, $\delta_{n}<a_{0}-\mu$

Also $\delta_{0}>\xi_{0} \Longrightarrow \delta_{0}>g_{0}-\mu$

Hence, $g_{0}-\mu<\delta_{n}<a_{0}-\mu$, i.e., the sequence $\left\{\delta_{n}\right\}$ is bounded.

Hence, the sequence $\left\{\delta_{n}\right\}$ is convergent and converges to a point $\delta_{A G}$ in $\left(g_{0}-\mu, a_{0}-\mu\right)$.

Accordingly, $\left\{a_{n}\right\} \&\left\{g_{n}\right\}$ and hence $A G M\left(x_{1}, x_{2}, \ldots \ldots, x_{N}\right)$ converges to a point $\mu+\delta_{A G}$.

Therefore, $\operatorname{AGM}\left(x_{1}, x_{2}, \ldots . ., x_{N}\right)$ can be regarded as a measure of the value of $\mu$ and consequently the central tendency of the values $\left(x_{1}, x_{2}, \ldots . ., x_{N}\right)$ with deviation $\delta_{A G}$ in $\left(g_{0}-\mu, a_{0}-\mu\right)$.

\subsection{AHM as a Measure of Central Tendency}

Since each of $a_{n}^{\prime} \& h_{n}^{\prime}$ is approximate value of $\mu$,

$a_{0}^{\prime}=\mu+\delta_{0}^{\prime}$ and $h_{0}^{\prime}=\mu+e_{0}^{\prime}$, for real numbers $\delta_{0}^{l}$ and $e_{0}^{\prime}$.

This implies, $\delta_{0}^{l}>e_{0}^{l}$ since $a_{0}^{l}>h_{0}^{l}$.

By the same logic,

$a_{n+1}^{\prime}=\mu+\delta_{n+1}^{\prime}$ and $h_{n+1}^{\prime}=\mu+e_{n+1}^{\prime}$, for real numbers $\delta_{n+1}^{\prime}$ and $e_{n+1}^{\prime}$.

Since $a_{n+1}^{\prime}$ is the AM of $a_{n}^{\prime}$ and $h_{n}^{\prime}$

Therefore, $a_{n}^{\prime}>a_{n+1}^{\prime}>h_{n}^{\prime}$

Which implies, $\delta_{n}^{\prime}>\delta_{n+1}^{\prime}$, i.e., sequence $\left\{\delta_{n}^{/}\right\}$is decreasing.

Moreover, $h_{0}-\mu<\delta_{n}^{\prime}<a_{0}-\mu$

i.e, the sequence $\left\{\delta_{n}^{\prime}\right\}$ is bounded

Hence, the sequence $\left\{\delta_{n}^{h}\right\}$ is convergent and converges to a point $\delta_{A H}$ in $\left(h_{0}-\mu, a_{0}-\mu\right)$. Accordingly, $\left\{a_{n}^{\prime}\right\} \&\left\{h_{n}^{\prime}\right\}$ and hence $A H M\left(x_{1}, x_{2}, \ldots . ., x_{N}\right)$ converges to a point $\mu+\delta_{A H}$.

Therefore, $A H M\left(x_{1}, x_{2}, \ldots . ., x_{N}\right)$ can be regarded as a measure of the value of $\mu$ and consequently the central tendency of the values $\left(x_{1}, x_{2}, \ldots . ., x_{N}\right)$ with deviation $\delta_{A H}$ in $\left(h_{0}-\mu, a_{0}-\mu\right)$.

\subsection{GHM as a Measure of Central Tendency}

Since each of $g_{0}^{/ /}=g_{0} \& h_{0}^{/ /}=h_{0}$ is approximate value of $\mu$, $g_{0}^{/ /}=\mu+\xi_{0}$ and $h_{0}^{/} /=\mu+e_{0}$, for real numbers $\xi_{0}$ and $e_{0}$.

This implies, $\xi_{0}>e_{0}$ since $g_{0}^{/ /}>h_{0}^{/ /}$.

By the same logic,

$g_{n+1}^{/ /}=\mu+\xi_{n+1}^{/ /}$and $h_{n+1}^{/ /}=\mu+e_{n+1}^{/ /}$, for real numbers $\xi_{n+1}^{/ /}$and $e_{n+1}^{/ /}$.

Since $g_{n+1}^{/ /}$is the GM of $g_{n}^{/ /}$and $h_{n}^{/ /}$

Therefore, $g_{n}^{/ /}>g_{n+1}^{/ /}>h_{n}^{/ /}$

Which implies, $\xi_{n}^{/ /}>\xi_{n+1}^{/ /}$, i.e., sequence $\left\{\xi_{n}^{/ /}\right\}$is decreasing.

Moreover, $h_{0}-\mu<\xi_{n}^{\prime /}<g_{0}-\mu$

i.e, the sequence $\left\{\xi_{n}^{/ /}\right\}$is bounded

Hence, the sequence $\left\{\xi_{n}^{/ /}\right\}$is convergent and converges to a point $\xi_{G H}$ in $\left(h_{0}-\mu, g_{0}-\mu\right)$. Accordingly, $\left\{g_{n}^{/ /}\right\} \&\left\{h_{n}^{/ /}\right\}$and hence $\operatorname{GHM}\left(x_{1}, x_{2}, \ldots . ., x_{N}\right)$ converges to a point $\mu+\xi_{G H}$.

Therefore, $G H M\left(x_{1}, x_{2}, \ldots . ., x_{N}\right)$ can be regarded as a measure of the value of $\mu$ and consequently the central tendency of the values $\left(x_{1}, x_{2}, \ldots . ., x_{N}\right)$ with deviation $\xi_{G H}$ in $\left(h_{0}-\mu, g_{0}-\mu\right)$. 


\subsection{AGHM as a Measure of Central Tendency}

In this case as earlier,

$a_{0}^{/ / /}=\mu+\delta_{0}, g_{0}^{/ / /}=\mu+\xi_{0} \& h_{0}^{/ / /}=\mu+e_{0}$ is approximate value of $\mu$,

$g_{0}^{/ /}=\mu+\xi_{0}$ and $h_{0}^{\prime} /=\mu+e_{0}$, for real numbers $\delta_{0}, \xi_{0}$ and $e_{0}$.

This implies, $\delta_{0}>\xi_{0}>e_{0}$ since $a_{0}^{/ / /}>g_{0}^{/ / /}>h_{0}^{/ / /}$.

By the same logic,

$a_{n+1}^{/ / /}=\mu+\delta_{n+1}^{/ / /}, g_{n+1}^{/ / /}=\mu+\xi_{n+1}^{/ / /}, h_{n+1}^{/ / /}=\mu+e_{n+1}^{/ / /}$for real numbers $\delta_{n+1}^{/ /}, \xi_{n+1}^{/ / /}$and $e_{n+1}^{/ / /}$.

Since $a_{n+1}^{/ / /}$is the AM of $a_{n}^{/ / /}, g_{n}^{/ / /}$and $h_{n}^{/ / /}$

Therefore, $a_{n}^{/ / /}>a_{n+1}^{/ / /}>h_{n}^{/ / /}$

Which implies, $\delta_{n}^{/ / /}>\delta_{n+1}^{/ / /}$, i.e., sequence $\left\{\delta_{n}^{/ / /}\right\}$is decreasing.

Moreover, $h_{0}-\mu<\delta_{n}^{/ / /}<a_{0}-\mu$

i.e, the sequence $\left\{\delta_{n}^{/ / /}\right\}$is bounded

Hence, the sequence $\left\{\delta_{n}^{/ / /}\right\}$is convergent and converges to a point $\delta_{A G H}$ in $\left(h_{0}-\mu, a_{0}-\mu\right)$. Accordingly, the sequences $\left\{a / h_{n}^{/ /}\right\},\left\{g_{n}^{/ / / /}\right\} \&\left\{h_{n}^{/ /}\right\}$and hence $A G H M\left(x_{1}, x_{2}, \ldots \ldots, x_{N}\right)$ converges to a point $\mu+\xi_{G H}$. Therefore, $G H M\left(x_{1}, x_{2}, \ldots . ., x_{N}\right)$ can be regarded as a measure of the value of $\mu$ and consequently the central tendency of the values $\left(x_{1}, x_{2}, \ldots ., x_{N}\right)$ with deviation $\delta_{A G H M}$ in $\left(h_{0}-\mu, a_{0}-\mu\right)$.

\section{Numerical Example - Application to Numerical data}

Observed data considered here are the data on each of annual maximum \& annual minimum of surface air temperature, occurred in temperature periodic year (TPR), at Guwahati during the period from 1969 to 2013 [Chakrabarty $(2014 \mathrm{c}, 2015 \mathrm{a}, 2015 \mathrm{c}, 2015 \mathrm{~d}, 2015 \mathrm{e}, 2015 \mathrm{f})]$. The objective here is to evaluate the value of central tendency of each of annual maximum \& annual minimum of surface air temperature at Guwahati.

\subsection{Central Tendency of Annual Minimum of Surface Air Temperature at Guwahati}

From the observed data on annual maximum of surface air temperature, occurred in temperature periodic year (TPR), at Guwahati during the period from 1969 to 2013, values (in Degree Celsius) of the seven measures of central tendency have been found as follows:

$$
\begin{aligned}
& A M=37.2093023255814, \\
& G M=37.1922871485760 \\
& H M=37.17539890356262 \\
& A G M=37.20079425067069371656824015813 \\
& A H M=37.188111479222283218438295127449 \\
& G H M=37.183841587880081504883830979786 \\
& A G H M=37.192326883785690452815011297444
\end{aligned}
$$

\subsection{Central Tendency of Annual Minimum of Surface Air Temperature at Guwahati}

From the observed data on annual minimum of surface air temperature, occurred in temperature periodic year (TPR), at Guwahati during the period from 1969 to 2013, values (in Degree Celsius) of the seven 
measures of central tendency have been found as follows:

$A M=7.3634146341463414634146341463415$,

$$
\begin{aligned}
& G M=7.2597176194576185608709616351297, \\
& H M=7.1543933802823525209849744707569, \\
& A G M=7.3114742070301664641236221835825, \\
& A H M=7.258151618339946610217427950892, \\
& G H M=7.2067668951373700073793727700802, \\
& A G H M=7.2586735571288657555393158774538
\end{aligned}
$$

\section{Conclusion}

From the description, presented above, one can arrive at the following conclusions:

1. In addition to AM, GM, \& HM, each of the four formulations namely AGM, AHM, GHM \& AGHM can be regarded as a measure of average of a set of numbers.

2. AGM, AHM, GHM \& AGHM are defined only when the associated numbers are strictly positive. For numbers other than strictly positive, there is necessity of searching for technique of finding these types of average.

3. Each of AGM, AHM, GHM \& AGHM can be accepted as measure of central tendency of data in addition to its usual measures namely AM, GM, \& HM.

4. Each of AM, GM, \& HM becomes closer and closer to the value of the central tendency of data if the data size becomes larger and larger. However, for data of small size, if is the difference among AM, GM, \& HM is found to be unacceptable then AGM, AHM, GHM \& AGHM may be preferred in finding the value of central tendency of data.

From the meaning of research [Chakrabarty, 2018g,h, 2019f], it can be concluded that the derivation of AGM, AHM, GHM \& AGHM as measures of central tendency of data can be regarded as research findings carrying fundamental importance and high significance in the theory of analysis of data specially of measure of central tendency of data..

\section{References}

Bakker, A. (2003). The early history of average values and implications for education. Journal of Statistics Education, 11(1).

Chakrabarty, D. (2014a). Analysis of errors associated to observations of measurement type. International Journal of Electronics and Applied Research, 1(1):15-28. https://doi.org/10.33665/IJEAR.2014.v01i01.001.

Chakrabarty, D. (2014b). Determination of parameter from observations composed of itself and errors. International Journal of Engineering Science and Innovative Technology, 3(2):304-311.

Chakrabarty, D. (2014c). Observation composed of a parameter and chance error : an analytical method of determining the parameter. International Journal of Electronics and Applied Research, 1(2):20-38. https://doi.org/10.33665/IJEAR.2014.v01i02.001.

Chakrabarty, D. (2015a). Central tendency of annual extremum of surface air temperature at guwahati. Journal of Chemical, Biological and Physical Sciences. Sec. C, 5(3):2863 - 2877. 
Chakrabarty, D. (2015b). Central tendency of annual extremum of surface air temperature at guwahati based on midrange and median. Journal of Chemical, Biological and Physical Sciences. Sec. D, $5(3): 3193-3204$.

Chakrabarty, D. (2015c). A method of finding true value of parameter from observation containing itself and chance error. Indian Journal of Scientific Research and Technology, 3(4):14-21.

Chakrabarty, D. (2015d). Observation composed of a parameter and chance error : an analytical method of determining the parameter. International Journal of Electronics and Applied Research, 2(1):35 47. https://doi.org/10.33665/IJEAR.2015.v02i01.001.

Chakrabarty, D. (2015e). Observation composed of a parameter and chance error : an analytical method of determining the parameter. International Journal of Electronics and Applied Research, 2(2):29 45. https://doi.org/10.33665/IJEAR.2015.v02i01.001.

Chakrabarty, D. (2015f). Observation consisting of parameter and error : determination of parameter. In Proceedings of the World Congress on Engineering, volume 2.

Chakrabarty, D. (2015g). Observation consisting of parameter and error: Determination of parameter. Lecture Notes in Engineering and Computer Science (ISBN: 978-988-14047-0-1), London,680-684.

Chakrabarty, D. (2016a). Impact of error contained in observed data on theoretical model : study of some important situations. International Journal of Advanced Research in Science, Engineering and Technology, 3(1):1255-1265.

Chakrabarty, D. (2016b). Pythagorean mean : concept behind the averages and lot of measures of characteristics of data. Paper presented in National Seminar on Advances in Electronics and Allied Science \& Technology, 2016, held in Gauhati Univerity, India,Abstract ID : CMAST-NaSAEAST-1601(Inv).

Chakrabarty, D. (2017a). Chakrabarty dhritikesh, variable(s) connected by theoretical model and model for respective observed data. Paper presented in FSDM2017,Abstract ID: FSDM 2220.

Chakrabarty, D. (2017b). Numerical data containing one parameter and random error : evaluation of the parameter by convergence of statistic. International Journal of Electronics and Applied Research, 4(2):59 - 73. https://doi.org/10.33665/IJEAR.2017.v04i02.001.

Chakrabarty, D. (2017c). Theoretical model and model satisfied by observed data: One pair of related variables. International Journal of Advanced Research in Science, Engineering and Technology, $3(2): 1527-1534$.

Chakrabarty, D. (2018a). Derivation of some formulations of average from one technique of construction of mean. American Journal of Mathematical and Computational Sciences, 3(3):62-68.

Chakrabarty, D. (2018b). $f_{h}-$ mean : one generalized definition of average. Journal of Environmental Science, Computer Science and Engineering \& Technology, Section C, 7(4):301-314.

Chakrabarty, D. (2018c). General technique of defining average. Paper presented in National Seminar on Advances in Electronics and Allied Science \& Technology, 2018, held in Gauhati Univerity, India,Abstract ID : CMAST-NaSAEAST-1801(Inv).

Chakrabarty, D. (2018d). Generalized $f_{g}-$ mean : derivation of various formulations of average. American Journal of Computation, Communication and Control, 5(3):101-108.

Chakrabarty, D. (2018e). Observed data containing one parameter and random error : evaluation of the parameter applying pythagorean mean. International Journal of Electronics and Applied Research, 5(1):32 - 45. https://doi.org/10.33665/IJEAR.2018.v05i01.001.

Chakrabarty, D. (2018f). One generalized definition of average : derivation of formulations of various means. Journal of Environmental Science, Computer Science and Engineering \& Technology, 7(3):212225 . 
Chakrabarty, D. (2018g). Statistics and bioscience : association in research. Significances of Bioengineering \& Biosciences, 2(5):001-007.

Chakrabarty, D. (2018h). Understanding the space of research. Biostatistics and Biometrics Open Access Journal, 4(4):87-93. DOI: 10.19080/BBOAJ.2018.04.555642.

Chakrabarty, D. (2019a). Association of statistics with biostatistics research. Biometrics \& Biostatistics International Journal, 8(3):104-109.

Chakrabarty, D. (2019b). A general method of defining average of function of a set of values. Aryabhatta Journal of Mathematics \& Informatics, 11(2):269-284.

Chakrabarty, D. (2019c). Observed data containing one parameter and random error: probabilistic evaluation of parameter by pythagorean mean. International Journal of Electronics and Applied Research, 6(1):24 - 40. https://doi.org/10.33665/IJEAR.2018.v06i01.001.

Chakrabarty, D. (2019d). One definition of generalized $f_{g}-$ mean : derivation of various formulations of average. Journal of Environmental Science, Computer Science and Engineering $\mathcal{G}$ Technology, Section-C, 8(2):51-66.

Chakrabarty, D. (2019e). One general method of defining average : derivation of definitions formulations of various means. Journal of Environmental Science, Computer Science and Engineering $\& 3$ Technology, Section-C, 8(4):327-338.

Chakrabarty, D. (2019f). Pythagorean geometric mean : measure of relative change in a group of variables. Abstract ID: CMAST-NaSAEAST-1902(Inv), Paper presented in National Seminar on Advances in Electronics and Allied Science \& Technology, 2019, held in Gauhati University, India.

Chakrabarty, D. (2020a). Ahm: a measure of the value of parameter $\mu$ of the model $x=\mu+e$. International Journal of Advanced Research in Science, Engineering and Technology, 7(10):1526815276.

Chakrabarty, D. (2020b). Arithmetic-harmonic mean : evaluation of parameter from observed data containing itself and random error. International Journal of Electronics and Applied Research, 7(1):2945. https://doi.org/10.33665/IJEAR.2020.v07i01.001.

Chakrabarty, D. (2020c). Central tendency of annual extremum of surface air temperature at guwahati by aghm. International Journal of Advanced Research in Science, Engineering and Technology, 7(12):16088-16098.

Chakrabarty, D. (2020d). Definition/formulation of average from first principle. Journal of Environmental Science, Computer Science and Engineering ${ }_{6} 3$ Technology, Section - C, 9(2):151-163.

Chakrabarty, D. (2020e). Determination of the value of parameter $\mu$ of the model $x=\mu+e$ by ghm. International Journal of Advanced Research in Science, Engineering and Technology, 7(11):1580115810.

Chakrabarty, D. (2020f). AGM: a technique of determining the value of parameter from observed data containing itself and random error. Journal of Environmental Science, Computer Science and Engineering E3 Technology, Section - C, 9(3):473-486.

Chakrabarty, D. (2021a). Aghm as a tool of evaluating the parameter from observed data containing itself and random error. International Journal of Electronics and Applied Research, 7(2):05 - 23. https://doi.org/10.33665/IJEAR.2020.v07i02.001.

Chakrabarty, D. (2021b). Agm, ahm, ghm \& aghm: evaluation of parameter $\mu$ of the model $x=\mu+e$. International Journal of Advanced Research in Science, Engineering and Technology, 8(2):16691-16699.

Chakrabarty, D. (2021c). Ahm as a measure of central tendency of sex ratio. Biometrics \& Biostatistics International Journal, 10(2):50-57. DOI: 10.15406/bbij.2021.10.00330. 
Chakrabarty, D. (2021d). Arithmetic-harmonic mean: a measure of central tendency of ratio-type data. International Journal of Advanced Research in Science, Engineering and Technology, 8(5):17324-17333.

Chakrabarty, D. (2021e). Comparison of measures of parameter of the model $X=\mu+e$ based on pythagorean means. International Journal of Advanced Research in Science, Engineering and Technology, 8(3):16948-16956.

Chakrabarty, D. (2021f). Formulation of average from pythagorean means: improved measure of central tendency of data. Paper presented in The 7th International Conference on Fuzzy Systems and Data Mining (FSDM 2021), held in Seoul, South Korea, Oct. 26-29,,Abstract ID: FSDM 3612.

Chakrabarty, D. (2021g). Four formulations of average derived from pythagorean means. International Journal of Mathematics Trends and Technology, 67(6):97-118.

Chakrabarty, D. (2021h). Model describing central tendency of data, international journal of advanced research in science. International Journal of Advanced Research in Science, Engineering and Technology, $8(9): 18193-18201$.

Chakrabarty, D. (2021i). Recent development on general method of defining average : a brief outline. International Journal of Advanced Research in Science, Engineering and Technology, 8(8):17947-17955.

David, A. (2004). Pi: A Source Book, chapter The Arithmetic-Geometric Mean of Gauss, pages 275-330. Springer, J. L. Berggren, Jonathan M. Borwein, Peter Borwein edition.

De Carvalho, M. (2016). Mean, what do you mean ? The American Statistician, 70(3):270274.

Foster, D. and Phillips, G. (1984). The arithmetic-harmonic mean. Mathematics of computation, 42(165):183-191.

Hazewinkel, M. (2001). Arithmetic-geometric mean process, Encyclopedia of Mathematics. Springer Science+Business Media B.V. / Kluwer Academic Publishers.

Kolmogorov, A. (1930). On the notion of mean, in mathematics and mechanics.

Kolmogorov, A. (1933). Grundbegriffe der Wahrscheinlichkeitsrechnung (in German). Julius Springer, Berlin.

Plackett, R. L. (1958). Studies in the history of probability and statistics : vii. the principle of the arithmetic mean. Biometrika, 45(1-2):130-135.

Riedweg, C. (2005). Pythagoras : his life, teaching, and influence. Cornell University Press. 0-8014$4240-0$.

Weisberg, H. and Weisberg, H. F. (1992). Central tendency and variability. Number 83. Sage.

Williams, R. (1984). Measures of central tendency. In Introduction to Statistics for Geographers and Earth Scientists, pages 51-60. Springer. 